$$
\begin{aligned}
& \operatorname{arcsec}(x)=\arccos \left(\frac{1}{x}\right), \\
& \operatorname{arccsc}(x)=\frac{\pi}{2}-\operatorname{arcsec}(x),
\end{aligned}
$$

(1) implies that each inverse trigonometric function is differentiable on the interior of its domain. Consequently, each derivative can then be computed by implicit differentiation or the chain rule.

\title{
Finding Matrices that Satisfy Functional Equations
}

Scott Duke Kominers (skominers@gmail.com), Harvard University

doi:10.4169/193113409X458769

Linear algebra is typically taught shortly after calculus. Thus, calculus-informed linear algebra problems offer an exceptional opportunity to illustrate interactions between different branches of undergraduate mathematics.

For example, consider the following problem, which (as we shall see) has its roots in calculus:

Problem. Find a $4 \times 4$, nonsingular, nonconstant matrix function $N(x)$ that satisfies the functional equation

$$
N(2 x)-(N(x))^{8}=0
$$

At first glance, this problem appears to be quite difficult. Beyond the likely difficulty of finding such a matrix $N(x)$, it is not even immediately clear how one would prove that a matrix $N(x)$ is actually a solution without a great deal of matrix algebra. However, this problem is not hard as it seems. In fact, it is one of a large class of problems that can be solved via a surprising method based upon single-variable calculus.

In this JOURNAL, Khan [2] used nilpotent matrices and Taylor series to find matrix functions satisfying the exponential functional equation, $f(x+y)=f(x) \cdot f(y)$. His method is an example of a much more general theory of matrix power series due to Weyr [4], which can be used to find matrix functions satisfying a variety of functional equations. (Rinehart [3] gives an excellent survey of Weyr's approach. Higham [1, ch. 4] gives a more comprehensive account, as well as further applications.)

We say that a set of real-valued functions $\left\{f_{i}(x)\right\}_{i=1}^{n} \subset \mathcal{C}^{\infty}(\mathbb{R})$ satisfies an analytic functional equation $E$ if there is an analytic function $E$ such that

$$
E\left(f_{1}, \ldots, f_{n}\right)(x)=0
$$

identically for all $x \in \mathbb{R}$. For example, the trigonometric functions $f_{1}(x)=\sin (x)$ and $f_{2}(x)=\cos (x)$ satisfy the analytic functional equation

$$
E\left(f_{1}, f_{2}\right)=f_{1}^{2}+f_{2}^{2}-1 \equiv 0 .
$$

Now, for any set of real-valued functions $\left\{f_{i}(x)\right\}_{i=1}^{n} \subset \mathcal{C}^{\infty}(\mathbb{R})$ satisfying the analytic functional equation $E$, we will find a set of associated matrix functions $\left\{A_{i}(x)\right\}_{i=1}^{n}$ satisfying the same functional equation $E$. 
Approximating each $f_{i}$ by its Taylor expansion about the origin, we obtain

$$
f_{i}(x)=f_{i}(0) x^{0}+\frac{f_{i}^{(1)}(0) x}{1 !}+\frac{f_{i}^{(2)}(0) x^{2}}{2 !}+\cdots+\frac{f_{i}^{(k)}(0) x^{k}}{k !}+\cdots,
$$

where $f_{i}^{(j)}$ is the $j$ th derivative of the function $f_{i}$. We let $A$ be any nilpotent matrix with index of nilpotence $k$ and then take

$$
\begin{aligned}
A_{i}(x):=f_{i}(A x) & =f_{i}(0) I+\frac{f_{i}^{(1)}(0) A x}{1 !}+\frac{f_{i}^{(2)}(0) A^{2} x^{2}}{2 !}+\cdots \\
& =f_{i}(0) I+\frac{f_{i}^{(1)}(0) A x}{1 !}+\frac{f_{i}^{(2)}(0) A^{2} x^{2}}{2 !}+\cdots+\frac{f_{i}^{(k-1)}(0) A^{k-1} x^{k-1}}{(k-1) !} .
\end{aligned}
$$

If the functions $\left\{f_{i}(x)\right\}_{i=1}^{n}$ satisfy the analytic functional equation $E$, then the Taylor series of the $f_{i}$ do as well, as $E$ is continuous. Thus, the matrix functions $\left\{A_{i}(x)\right\}_{i=1}^{n}$ found from the Taylor series of the $f_{i}$ must also satisfy the functional equation $E$.

We begin with a simple example. For aesthetic reasons, we will work with the nilpotent matrix

$$
A=\left(\begin{array}{llll}
0 & 0 & 12 & 0 \\
0 & 0 & 0 & 12 \\
6 & 6 & 0 & 0 \\
-6 & -6 & 0 & 0
\end{array}\right)
$$

We obtain from the Taylor series for $f_{1}=\sin (x)$ and $f_{2}=\cos (x)$ the matrices

$$
\begin{aligned}
& A_{1}(x)=f_{1}(A x)=A x-\frac{A^{3} x^{3}}{6}=\left(\begin{array}{llll}
0 & 0 & 12 x-144 x^{3} & -144 x^{3} \\
0 & 0 & 144 x^{3} & 144 x^{3}+12 x \\
6 x & 6 x & 0 & 0 \\
-6 x & -6 x & 0 & 0
\end{array}\right), \\
& A_{2}(x)=f_{2}(A x)=I-\frac{A^{2} x^{2}}{2}=\left(\begin{array}{llll}
1-36 x^{2} & -36 x^{2} & 0 & 0 \\
36 x^{2} & 36 x^{2}+1 & 0 & 0 \\
0 & 0 & 1-36 x^{2} & -36 x^{2} \\
0 & 0 & 36 x^{2} & 36 x^{2}+1
\end{array}\right) .
\end{aligned}
$$

We have immediately from this construction that $A_{1}(x)$ and $A_{2}(x)$ commute. More interestingly, these matrix functions satisfy the trigonometric functional equations. We therefore find the familiar identity

$$
\left(A_{1}(x)\right)^{2}+\left(A_{2}(x)\right)^{2}=I .
$$

Similarly, we obtain analogues of the "double-angle" formulas,

$$
\begin{aligned}
& A_{1}(2 x)=2 A_{1}(x) A_{2}(x), \\
& A_{2}(2 x)=\left(A_{2}(x)\right)^{2}-\left(A_{1}(x)\right)^{2}=2\left(A_{2}(x)\right)^{2}-I .
\end{aligned}
$$

Although the matrices found via this method need not be nonsingular, in general, the matrix $A_{2}(x)$ is, as $\operatorname{det} A_{2}(x)=1 \neq 0$. We can therefore invert $A_{2}(x)$ and obtain an analogue of the secant-tangent trigonometric square identity:

$$
\left(A_{2}^{-1}(x)\right)^{2}=I+\left(A_{1}(x) A_{2}^{-1}(x)\right)^{2} .
$$


As a second example of our approach, let us solve the problem stated at the beginning of this Capsule. We seek a $4 \times 4$, nonconstant matrix function $N(x)$ satisfying the functional equation

$$
N(2 x)-(N(x))^{8}=0 .
$$

As before, we consider the associated functional equation in nonconstant $\mathcal{C}^{\infty}(\mathbb{R})$ functions,

$$
g(2 x)-(g(x))^{8}=0 .
$$

Now, this condition immediately implies that either $g(0)=0$ or $(g(0))^{7}=1$. In the former case, $g$ would vanish to some order $k>0$ at the origin, and we would then have $k=8 k$ from the functional equation-impossible. Thus, $g(0)$ is a seventh root of unity, and we may require $g(0)=1$ by considering the function $g / g(0)$ if necessary.

Then, we write $g(x)=1+g_{n} x^{n}+\cdots$ for some minimal $n>0$ and $g_{n} \neq 0$. By the functional equation, we must have

$$
1+2^{n} g_{n} x^{n}+\cdots=g(2 x)=(g(x))^{8}=\left(1+g_{n} x^{n}+\cdots\right)^{8}=1+8 g_{n} x^{n}+\cdots .
$$

Equating coefficients on both sides then gives that $n=3$; we may also assume that $g_{3}=1$ by replacing $x$ by $x / \sqrt[3]{g_{n}}$. If we now write $g(x)=1+x^{3}+g_{m} x^{m}+\cdots$, for $m>3$ minimal and $g_{m} \neq 0$, we obtain from a further application of the functional equation that $m=6$. Thus, for some $g_{6} \neq 0$,

$$
g(x)=1+x^{3}+g_{6} x^{6}+\cdots .
$$

Since the matrix $A$ chosen above has index of nilpotence $4<6$, we have found sufficient information to compute the matrix $N(x):=g(A x)$. Indeed, we have

$$
\begin{aligned}
N(x)=g(A x)=I+A^{3} x^{3}+g_{6} A^{6} x^{6}+\cdots & =I+A^{3} x^{3} \\
& =\left(\begin{array}{llll}
1 & 0 & 864 x^{3} & 864 x^{3} \\
0 & 1 & -864 x^{3} & -864 x^{3} \\
0 & 0 & 1 & 0 \\
0 & 0 & 0 & 1
\end{array}\right) .
\end{aligned}
$$

It is immediate from this construction that $N(x)$ satisfies the desired functional equation.

Unwinding our technique, we obtain a general approach to problems that ask for matrices satisfying analytic functional equations. Specifically, one can approach a problem asking for matrices $\left\{A_{i}(x)\right\}_{i=1}^{n}$ satisfying an analytic equation

$$
E\left(A_{1}(x), \ldots, A_{n}(x)\right) \equiv 0
$$

by trying to solve the equation $E$ for real-valued functions $\left\{f_{i}(x)\right\}_{i=1}^{n}$. If any of these solutions $\left\{f_{i}(x)\right\}_{i=1}^{n}$ satisfy $f_{i} \in \mathcal{C}^{\infty}(\mathbb{R})$ for all $i$, then it is possible to find matrix solutions $\left\{A_{i}(x)\right\}_{i=1}^{n}$ of any dimension $n$ by applying our method with an $n \times n$ nilpotent matrix.

This approach demonstrates a surprising connection between calculus and linear algebra. It could also serve as an elementary introduction to the theory of functions of matrices.

Acknowledgments. The author is grateful to Zachary Abel, Andrea Hawksley, and several anonymous referees for their helpful suggestions on earlier drafts of this article. He especially thanks Noam D. Elkies for his comments regarding the second example. 


\section{References}

1. N. J. Higham, Functions of Matrices: Theory and Computation, SIAM, 2008.

2. M. A. Khan, A class of exponential matrices, this Journal 34 (2003) 194-195.

3. R. F. Rinehart, The equivalence of definitions of a matrix function, Amer. Math. Monthly 62 (1955) 395-414.

4. E. Weyr, Note sur la théorie de quantités complexes formées avec n unites principales, Bull. Sci. Math. II 11 (1887) 205-215.

\section{Teaching Tip: The Median Is a Balance Point}

\section{Mark Lynch (lynchmj@millsaps.edu), Millsaps College, Jackson MS 39210}

It is well-known that the mean of a data set $\left\{x_{1}, x_{2}, \ldots, x_{n}\right\}$ can be interpreted as a balance point, in the sense that if these points are marked along a uniform rod and equal weights are placed at each of these locations, then a fulcrum must be placed at the mean of the data in order for the rod to balance. The reason for this is that the sum of the torques must be zero for the rod to balance. The equation to be solved is $\sum\left(x_{i}-p\right) w=0$, where $p$ is the location of the fulcrum and $w$ is the weight placed at $x_{i}$ (see Figure 1(a)). Solving for $p$, we get $p=\sum x_{i} / n$, the mean of the data.

A balancing procedure for the median of a data set is not known. Over the years, many students have commented that this gives the mean 'bragging rights', since nature seems to have chosen it over the median. We now describe such a balancing procedure, and so give the median some well deserved street-cred in the classroom.

Replace the rod with a string, but keep the data markings and weights. Then, at the ends, attach a second string, longer than the first, to form a loop, and drape the loop over a well-lubricated pulley (see Figure 1(b, c)).

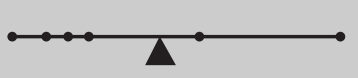

mean

(a)

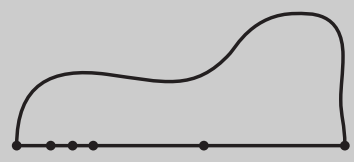

(b)

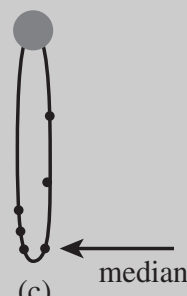

(c)

Figure 1

Suppose, initially, that the weights are distinct. The pulley and loop balance when the same number of weights are to each side. In other words, the loop 'balances' when the median is the lowest point. Since the second string is longer than the first, no weight is pulled over the pulley as the system rights itself. Notice that if one of the weights is slid way up the loop creating an outlier, the loop doesn't move. This demonstrates, physically, the principle that the median is unaffected by outliers.

When we have repeated values, it's possible for a cluster of weights to appear at the bottom of the loop and fewer weights on one side than the other (these fewer weights can't pull the cluster higher). But the lowest point of the loop is still the median in this case. When there are an even number of distinct points, the median is any point between the two middle values. We don't restrict our median to be the average of these middle values, since the pulley and loop need not balance at this midpoint. 